\title{
Behavioral genetics
}

The Cambridge Handbook of Evolutionary Perspectives on Sexual Psychology

Severi Luoto, University of Auckland

s.luoto@auckland.ac.nz

Michael A. Woodley of Menie, Center Leo Apostel for Interdisciplinary

Studies, Vrije Universiteit Brussel, Brussels, Belgium

Cite this chapter as:

Luoto, S. \& Woodley of Menie, M. A. (in press). Behavioral genetics. In T. Shackelford (Ed.), The Cambridge Handbook of Evolutionary Perspectives on Sexual Psychology. Cambridge University Press.

Word count: 12019 (body text only), 15352 (abstract, body text, references)

Keywords: behavioral genetics, evolutionary psychology, evolution, natural selection, sexual selection 


\begin{abstract}
In this introductory chapter, we discuss the nexus between evolutionary theory and behavioral genetics, using it to elucidate the biological origins of human behavior and motivational predispositions. We introduce relevant behavioral genetics methods and evolutionary theoretical background to provide readers with the necessary conceptual tools to deepen their engagement with evolutionary behavioral genetics - as well as to help them take on the challenge of building a scientifically and evolutionarily more consilient account of human behavior. To demonstrate the utility of behavioral genetics in evolutionary behavioral science, our analytical examples range from personality, cognition, and sexual orientation to pair-bonding. We conclude by presenting a few recent landmark studies in behavioral genetics research with a particular focus on two aspects of sexual behavior: assortative mating and same-sex sexual behavior. This chapter considers behavioral genetics methods and their connection with evolutionary science more broadly while providing a succinct overview of recent advances in understanding the evolutionary genetic underpinnings of human sexual behavior, mate choice, and basic motivational processes. It is a sine qua non of scientifically principled evolutionary behavioral scientists to acknowledge the distal evolutionary and proximal genetic processes which, interlinked, underlie the psychobehavioral predispositions that form the variegated fabric of human societies and, more broadly, the diversity of life found in nature. These evolutionary processes operate from distal selection pressures acting on genetic material through hundreds of millions of years of natural selection - and from individual and population differences in genotypes to their manifestations in complex behavioral phenotypes and life outcomes in contemporary humans - which, in turn, enact concomitant selection pressures on the genetic material underlying and arising from them.
\end{abstract}




\section{Introduction}

Evolutionary biology seeks to understand the ways in which various forms of selection make species better able to execute adaptations in order to maximise fitness (gene-copying success) in their varying environments. Evolutionary biology also studies non-adaptive evolutionary processes, which include genetic drift, mutation, and migration.

When Charles Darwin proposed the rudiments of what would become the theory of evolution by natural selection, it was not properly integrated with an understanding of genetics or with Mendel's laws of inheritance, neither of which were known when Darwin published On the Origin of Species by Means of Natural Selection in 1859. This integration occurred in the early $20^{\text {th }}$ century with the "modern synthesis" which arose from the work of Huxley, Dobzhansky, Fisher, Haldane, Wright, and Mayr. Since then, advances in genetics have been integral to empirical and theoretical developments in evolutionary behavioral science. Evolutionary behavioral science and evolutionary psychology have, for instance, increasingly included insights on genetic variation in models for population and individual differences in life history strategies, male and female sexual orientation, and various forms of selection for attractiveness, intelligence, and creativity.

Behavioral genetics is one of several fields with the potential to contribute to a sophisticated and mature science of evolutionary psychology. Behavioral genetics focusses on the elucidation of the genetic and environmental co-determinants of an organism's behavior and, more generally, on such influences on individual differences in behavior. Both sociobiology (the major precursor to modern evolutionary psychology) and behavioral genetics matured as fields in the 1970s, aiming to integrate the study of human behavior with other branches of biology; however, behavioral genetics and (what went on to become) evolutionary psychology have mostly developed in isolation (cf. Segal \& Macdonald, 1998; Zietsch, de Candia, \& Keller, 2015).

This distance between the fields arises from the way in which behavioral genetics focusses on proximate mechanisms - that is, how do genetic and environmental factors influence behavioral differences between individuals, and which specific genetic variants or environmental factors underlie individual differences in behavioral phenotypes. Evolutionary psychology, on the other hand, focusses (with some exceptions) on the ultimate level of explanation: what adaptive advantages, if any, do traits and behaviors provide for an organism, and what can this tell us about the relevant selection histories that shaped these adaptations in humans (Barkow, Cosmides, \& Tooby, 1992; Zietsch et al., 2015; Zietsch, Sidari, Murphy, Sherlock, \& Lee, 2020). Another point of divergence concerns the nature of the systems that each field specializes in studying. Behavioral genetics focusses on systems that vary between individuals (such as personality and intelligence), whereas the focus of evolutionary psychology has historically been on human universals: species-typical cognitive adaptations, or modules, that arose in response to specific, recurrent selection pressures in the course of human evolutionary history. Evolutionary psychologists have historically rejected 
the idea that such systems exhibit heritability as modules are species-typical, and should not significantly vary genetically among (normal) individuals. Any variation that might arise in relation to the functioning of these modules is expected to be essentially random and nonheritable, stemming from developmental miscalibration (Tooby \& Cosmides, 1990; see Woodley of Menie \& Sarraf, 2021 for a detailed discussion of the history of evolutionary psychology). Contemporary evolutionary psychology does, however, acknowledge the heritability of individual differences as well as the many ways in which environmental inputs, particularly during sensitive periods in development, shape human cognition and behavior (Ellis et al., 2020; Figueredo, Vasquez, Brumbach, \& Schneider, 2004; Luoto, Krams, \& Rantala, 2019a; Syme \& Hagen, 2020).

Many research paradigms that are adjacent to evolutionary psychology, such as personality and individual differences, developmental and behavioral neuroscience, and behavioral genetics, need to be better integrated with an evolutionary perspective in order to acquire a broader understanding of human cognition and behavior. To that end, there have been several attempts to synthesize the proximate and ultimate levels of analyses of individual differences and their determinants into what can broadly be termed "evolutionary psychology" (Lewis, Conroy-Beam, Asao, \& Buss, 2017; Luoto et al., 2019a; Rantala, Luoto, Borráz-León, \& Krams, 2021). Woodley of Menie and Sarraf (2021) have termed this "Broad Church" evolutionary psychology, so as to distinguish it from the more narrowly focussed "High Church" or Santa Barbara School evolutionary psychology (this term was coined by Heyes, 2012). This integration between proximate mechanisms and ultimate functions is a major goal to which a scientifically well-rounded and robust evolutionary behavioral science should aspire (Lewis et al., 2017; Tinbergen, 1963; Zietsch et al., 2020).

A major effort in this area has been to shift the focus of evolutionary psychology towards better understanding the ultimate functions and proximate mechanisms underlying individual differences. Personality, and more broadly life history variation (Woodley of Menie, Luoto, Peñaherrera-Aguirre, \& Sarraf, 2021), for example, might produce enough behavioral polymorphism that it can hedge against fluctuations in the levels of environmental risk and in the availability of environmental micro-niches (Figueredo et al., 2005; Wilson, 1998; Woodley, 2011; Young, Frankenhuis, \& Ellis, 2020). Individual differences might also arise from the sensitivity of cognitive and other systems to the (mostly deleterious) impacts of pleiotropic mutations (i.e., genetic variants that influence multiple systems) on their development. Thus, individual differences with respect to certain sources of both trait variation and covariation might arise from the action of various forms of selection on phenotypic condition, with this serving as a hard-to-fake signal of underlying system integrity (see e.g., Houle, 2000; Miller, 2000 on the possible existence of a "general fitness factor" among covarying sources of individual differences). Also significant are individual differences arising from the interaction between evolved psychological mechanisms and the environment (e.g., Del Giudice, 2020; Rantala, Luoto, Krama, \& Krams, 2019; Varella, Luoto, Soares, \& Valentova, 2021). Such individual differences might arise via a process of differential adaptive calibration in which the execution of biological and psychobehavioral adaptations is fine-tuned by virtue of specific environmental experiences early in 
development, giving rise to variation and covariation among personality and life history characteristics (Ellis \& Del Giudice, 2018; Frankenhuis \& Panchanathan, 2011; Luoto et al., 2019a; Woodley of Menie, Luoto, et al., 2021; Young et al., 2020). Other related ways in which such individual differences can arise include psychobiological mechanisms that produce variation in important phenotypic traits such as stress reactivity, eating behaviors, mental health, and sexual behaviors and preferences (Luoto et al., 2019a; Rantala et al., 2021, 2019; Syme \& Hagen, 2020).

Most human traits, such as intelligence, personality, psychopathology, and height, are substantially heritable (Crespi, 2020; Daniele, 2021; Gangestad, 2010; Krams et al., 2019; Penke \& Jokela, 2016; Plomin, DeFries, Knopik, \& Neiderhiser, 2016; Stulp \& Barrett, 2016). A meta-analysis of the heritability of all (studied) traits found that the aggregate heritability was 0.49 , meaning that $49 \%$ of the variance among all (studied) individual differences are due to the influence of genetic factors (Polderman et al., 2015). As Simon LeVay noted in his book, The Sexual Brain: "In reality, our range of individual development is defined and limited by what we are born with. Like waterlilies, we swing to and fro with the currents of life, yet our roots moor us each to our own spot on the river's floor" (LeVay, 1994, p. 138).

In this chapter, we focus on how behavioral genetics can be used in evolutionarily informed sex research. We first introduce the rationale of using behavioral genetic methods in evolutionary psychology (section 2), followed by a brief introduction to behavioral genetic methods (section 3). We then review specific case studies of salient examples of the use of behavioral genetic methodology in evolutionarily informed sex research (section 4), concluding this chapter in section 5 .

As the gene is the principal unit of selection, evolutionary behavioral scientists and evolutionary psychologists should make a serious effort to integrate behavioral genetics into their methodological toolkit. An evolutionary psychology that neglects evolutionary genetics is missing an important part of the biological puzzle (Keller, 2008).

\section{Behavioral genetics and evolutionary psychology}

The main ultimate-level question connected with behavioral genetics is: what evolutionary processes explain genetic variation? Specific proximate-level questions include: which genes underlie behavioral variation? What is the nature of the genetic variance underlying behavioral phenotypes? What physiological processes are involved in the pathway from genes to behavioral phenotypes? What gene-environment interactions shape behavioral phenotypes? (Gangestad, 2010). To understand these questions, we need to briefly discuss the processes by which selection leads to changes in allele frequencies over time and space - an integral component of evolutionary change. 
Table 1. Glossary of evolutionary behavioral genetics terminology. Adapted from Zietsch et al. (2015) and Luoto (2019a).

Allele: each of two or more alternative forms of a gene found in the same genetic locus. An allele segregates as a unitary Mendelian factor.

Assortative mating: a mating pattern and form of selection in which individuals with similar phenotypes mate with one another more frequently than would be expected by chance. In genetic assortative mating, individuals with similar genotypes mate with one another more frequently than would be expected by chance.

Broad-sense heritability: the percentage of variance in a given phenotype attributable to both additive and non-additive (i.e., dominance and epistasis) genetic effects.

Causal variant $(\mathbf{C V})$ : an allele that influences a trait.

Epigenetics: a field of science that studies changes caused by the activation and deactivation of genes. Unlike genetic changes, epigenetic changes are reversible and do not change the DNA sequence of an organism, although they can change how a DNA sequence is read.

Evolution: changes in organic forms stemming (at least in part) from changing allele frequencies across generations and over time and space. Microevolution is the process by which adaptive or non-adaptive individual and population differences within a species arise. Macroevolution explains evolutionary patterns above the species level, including the emergence of new species, genera, or other higher taxa. Although the majority of evolutionists see microevolution and macroevolution as intertwined, with the former necessarily yielding the latter, given enough time and opportunity, and genetic changes of one sort or another being sufficient to account for the origin of new species, this equivalence is nevertheless debated. Some prominent evolutionary theorists have argued that other processes, such as alterations in the patterns of structural constrains on organismal bodyplans, that are not easily reducible to mere adaptive genetic change, might be needed to facilitate macroevolution (e.g., Gould, 2002).

Fisher's fundamental theorem: "the rate of increase in fitness of any organism at any time is equal to its genetic variance in fitness at that time." It has often been interpreted to mean that additive genetic variation should be low in traits that are related to fitness.

Genome: all genetic material of an organism. The genome (also referred to as the genotype) contains all the information required to build and maintain that organism. There are two fundamental variants of the definition of genome: one refers to generic types of genes (genetic loci) and the other to specific types of genes (alleles). Genotype pertains specifically to the latter definition (Mahner \& Kary, 1997).

Genome-wide association study (GWAS): a test of associations between each of hundreds of thousands of single-nucleotide polymorphisms across the genome and one or more traits. Very large sample sizes are needed to detect the small effect sizes that are commonly reported across variants for complex polygenic traits. 
"Good" gene indicators: traits that are reflective of genetic factors that would have enhanced genetic fitness under the sorts of selection regimes that are believed to have characterized the human environment of evolutionary adaptedness, such as low mutation load.

Haplotype: a group of genes (a specific allelic composition) within an organism that are inherited together from a single parent.

Heritability: used synonymously with broad-sense heritability.

Heterozygosity: the presence of two different alleles at a genetic locus.

Homozygosity: the presence of two copies of the same allele at a genetic locus.

Linkage disequilibrium: the statistical relationship between alleles at different loci (positions in the genome), that is, the non-random association of alleles at different loci in a given population. Loci are considered to be in linkage disequilibrium when the frequency of association of their different alleles is higher or lower than would be expected if the loci were independent and associated randomly. Linkage disequilibrium can arise from physical linkage among alleles (i.e., where they are found together at specific locations in the chromosome), but can also exist among alleles at different loci without any physical linkage between them (i.e., epistasis) and independently of whether or not allele frequencies are in equilibrium (i.e., not changing with time) (for more information, see Slatkin, 2008). Linkage disequilibrium is also known as gametic phase disequilibrium.

Mutation load: the aggregate burden of (usually neutral or mildly) deleterious mutations (rare alleles) across the genome for an individual, which is heritable across generations.

Narrow-sense heritability: the proportion of genetic variation that is due to additive genetic factors only.

Natural selection: in evolutionary biology and evolutionary psychology, natural selection is commonly defined as the sum total of viability selection, sexual selection, and kin selection, that is, the differential heritable survival and reproduction of individuals carrying alleles on which different selection pressures operate (Cornwallis \& Uller, 2010; Proulx \& Østman, 2016; Wiley, 2020). There is some disagreement among evolutionists on how "natural selection" is defined, with some evolutionary ecologists (including one of the authors, MAWoM) preferring a definition whereby natural selection relates exclusively to extrinsic (i.e., uncontrollable) sources of mortality and morbidity that arise from the natural environment of the organism. These include biotic (such as predator-prey, or host-parasite dynamics) and physical (such as climatological or geological) factors, which contribute to shaping a species' adaptive landscape. Based on this definition, natural selection is distinct from sexual selection in that it solely refers to variation in fitness stemming from "hard" (i.e., environmental) rather than from "soft" (i.e., social) factors. Furthermore, natural selection pressures, based on this approach, provide the context in which sexual selection can operate in shaping adaptations (see e.g., Safran, Scordato, Symes, Rodríguez, \& Mendelson, 2013). Here, however, we follow the broader definition of natural selection commonly used in evolutionary biology and evolutionary psychology (Cornwallis \& Uller, 2010; Proulx \& Østman, 2016; Wiley, 2020), which encompasses viability selection, sexual selection, and kin selection. 
Phenotype: the composite of an organism's observable physical, cognitive, or biochemical traits or states (as determined by genetic makeup and environmental influences). Much literature exists on behavioral phenotypes; however, for reasons of theoretical accuracy and empirical distinction, behavioral traits can be distinguished from physical phenotypes (Luoto, 2019a).

Phenotypic plasticity: the ability of a single genotype to produce multiple phenotypes when exposed to different environments, often in adaptive ways.

Pleiotropy: when one gene influences two or more seemingly unrelated phenotypic traits, it is referred to as a pleiotropic gene.

Recessive/additive/dominant: the likelihood of an allele to be expressed as a phenotype. A fully recessive allele will not be expressed unless both copies are present at a diallelic locus. A dominant allele will be fully expressed with only one copy (or two in the case of codominant alleles). Many dominance relationships are partial rather than full, resulting in a spectrum of dominance and recessiveness. Variants with additive effects are intermediate between fully recessive and fully dominant, yielding gradations in trait variation.

Sexual selection: any variation in direct fitness among different phenotypes caused by their ability to gain sexual partners, produce fertilized eggs, and generate offspring.

SNP (single-nucleotide polymorphism): a type of allele where a single-nucleotide position is variable in the population. The term SNP is often used for loci where the minor allele frequency is $>1 \%$ and mutation when the minor allele frequency is $<1 \%$.

Viability selection: any variation in direct fitness among different phenotypes caused by their differential ability to survive (also referred to as survival selection).

Zygosity: the similarity or differences between DNA sequences on the chromosomes of a fertilized egg (i.e., "a zygote"); how genetically similar (or different) twins are. See also heterozygosity, homozygosity.

Every organism develops in a particular environment based on information encoded in its DNA, and as such, both its genotype and its phenotype are co-determined by its environment and its evolutionary history (Bateson \& Gluckman, 2011; Marciniak \& Perry, 2017; Rees, Castellano, \& Andrés, 2020; Wiley, 2020). Each lineage of organisms persists as long as it is able to harvest exogenous energy to counteract entropy and as long as its genes are able to make future copies of itself: each organism eventually decays, but its lineage persists if the organism has successfully transferred its genetic information to the next generation (see chapters 1 and 2 of this Handbook, and Ellison, 2017; Wiley, 2020). For this reason, it is important for evolutionary psychologists to focus on the genetic basis of behavior, as it is genetic material that gives rise to the structure of life, including evolutionarily relevant behavioral predispositions on which various forms of selection operate. This process will operate given 1) that organisms transmit their genotype to progeny with some appropriate 
level of variability and continuity, 2) that morphological, physiological, and psychobehavioral traits vary among individuals and are partially heritable, and 3) that this variation confers different rates of survival and reproduction. Given these initial conditions, selection can yield a lineage of organisms with contextually favorable characteristics that enable them to survive and reproduce in their respective environments. In this way, evolution through natural selection acts to optimize the ways in which organisms come to better execute adaptations by increasing the degree to which features of an organism's phenotype better fit factors in their (selectively relevant) environments (Barkow et al., 1992; Marciniak \& Perry, 2017; Rees et al., 2020; see also Luoto, 2019a, 2019b).

\subsection{Processes underlying genetic variability}

Genetic variation in a population across generations (i.e., evolution) can be produced by genetic mutations, reshuffling of genes through sexual reproduction, different modes of selection (directional, stabilizing, disruptive, frequency-dependent selection etc.), genetic drift (random variation in gene frequencies stemming from stochastic processes, such as differential fixation via fluctuations in population size and founder events), and by migration to, emigration from, and admixture among distinct populations (clinal gene flow).

\subsubsection{Selection}

Selection can act when there is heritable variation in fitness. Natural selection can be divided into its various constituent components ${ }^{1}$ : viability selection, sexual selection, and kin selection, which often work together to shape adaptations in ways that are complementary (Cornwallis \& Uller, 2010; Safran et al., 2013; Van Doorn, Edelaar, \& Weissing, 2009). Selection can be further categorized based on ecological factors and the direction of selection. In directional selection, forms of a phenotype that are skewed relative to the mean are favored (either above or below a population mean); in stabilizing selection, intermediate forms of a phenotype are favored over extreme forms; in disruptive selection, phenotypes that are positively and negative skewed with respect to the population mean are favored over the intermediate ones; and in frequency-dependent selection, fitness is related to the frequency of a phenotype or genotype in a population (this can be positive or negative based on whether the genotype has a larger fitness payoff from being rare or common within a population). In mutation-selection balance, genetic variation arises from the balance between deleterious mutations arising at many loci, persisting due to their small effects on the phenotype, contributing to genetic load, and their eventual removal by negative (also termed purifying) selection.

\footnotetext{
${ }^{1}$ Though see Table 1 for an alternative definition of "natural selection". One of us (MAWoM) prefers the alternative definition where natural selection does not encompass, but does contextualise, sexual selection as a distinct source of fitness variance (see e.g., Safran et al., 2013; Van Doorn et al., 2009). One of us (SL) prefers the definition where natural selection includes viability selection, sexual selection, and kin selection (see e.g., Cornwallis \& Uller, 2010; Proulx \& Østman, 2016; Wiley, 2020).
} 
Negative selection is more efficient at eliminating additive or dominant deleterious alleles compared with recessive or partially recessive deleterious alleles, because selection generally either does not act on, or only acts weakly on, recessive or partially recessive alleles when heterozygous (Zietsch et al., 2015). Deleterious alleles yet to be eliminated by negative selection are more likely to be recessive than is expected by chance. Directional dominance occurs when deleterious alleles are more recessive and beneficial alleles more dominant, a situation that can, under certain circumstances, be used to infer selection (Zietsch et al., 2015).

According to Fisher's (1930) fundamental theorem of natural selection, the rate at which the mean fitness in a population increases due to selection at a given time is equal to the genetic variance in fitness. This equation was generalized by G. R. Price by partitioning changes in genotypes in a subsequent generation into the covariance between genes and environment. Price's equation posits that this principle can apply to any trait, including learning as well as genetic variation (Wiley, 2020). The mechanisms of heredity vary across a spectrum of stability, from the relative stability of the genome to the increasing flexibility of developmental switches, epigenesis, and learning and cultural evolution (Bateson \& Gluckman, 2011; Uchiyama, Spicer, \& Muthukrishna, 2020; Wiley, 2020). Even traits that are under strong selection show ubiquitous genetic variation in humans and nonhuman animals (Woodley of Menie, Fernandes, \& Hopkins, 2015; Zietsch et al., 2015). The existence of genetic variation in fitness-related traits contradicts the traditional interpretation of Fisher's fundamental theorem, and explaining the evolutionary basis of such widespread genetic variation in fitness-related traits is an important open question in biology (Zietsch et al., 2015).

It remains a major challenge to understand the evolutionary underpinnings of genetic variation in traits because, rather than selection acting on fitness with respect to any given trait in isolation, selection acts on total "net fitness" where causal variants (CVs, that is, alleles with causal effects on a phenotype) often affect multiple traits simultaneously and many CVs affect a given trait (that is, they have pleiotropic effects). Therefore, a trait's CVs can be under many different types and strengths of selection (Zietsch et al., 2015). For instance, genetic variants are not selected in isolation, but can "hitch a ride" on the coattails of a neighboring allele that can be positively or negatively selected (Arslan \& Penke, 2015). As genes and alleles are inherited in groups, the likelihood of inheriting a certain allele from a parent is partially dependent on which alleles are present in neighboring genetic loci. Two or more alleles that usually co-occur (i.e., are in "linkage disequilibrium") form a haplotype; however, they can have different, even opposing effects on fitness, and they cannot be selected independently until recombination breaks them apart (Arslan \& Penke, 2015). Long (unbroken) haplotypes indicate that a new mutation has been strongly selected in past generations: the neighboring alleles of a beneficial mutation in such a haplotype are carried along before recombination can break them apart (Arslan \& Penke, 2015). A hard sweep is an event in which a single haplotype including a selectively advantageous allele becomes 
more frequent. In a soft sweep, multiple haplotypes harboring advantageous mutations simultaneously rise in frequency.

\subsubsection{Mutations}

The process of DNA replication, which in the human genome involves about 6.2 billion base pairs, is not perfect, and the simple principle of probability of error in copying such a large swathe of DNA results in occasional errors in the copying process. These errors, known as mutations, are at the core of evolutionarily relevant genetic change as they introduce new genetic variants, and thus information, into the gene pool. As errors introduced into the structure of DNA, mutations that involve a single base (A, C, G, or T) are called point mutations, which commonly involve the substitution of one base for another in the sequence. Rarer (and typically more deleterious) forms of point mutation include the insertion of a new base, the deletion of an existing base, or the inversion of an existing base-pair. Alterations in larger numbers of base-pairs (involving deletions and insertions) are known as copy-number variation. Larger changes in base-pair organization at the chromosomal level (such as translocations, deletions, or duplications - the latter two known together as aneuploidy) can also occur. Point mutations are the most common and best understood mutations (Keller, 2008).

Mutations most often occur during the replication of DNA prior to cell division; however, the probability that a mutation occurs during the replication of a given base-pair of DNA is remarkably low-approximately two errors per billion base-pair copying event - and is highly consistent across eukaryotic lifeforms (Keller, 2008). The low error rate is consistent with intense selection for fidelity in gene duplication. The majority of mutations arise in nongermline cells (they are somatic), and therefore have limited evolutionary relevance as they are not transmitted to offspring, even if they can result in harmful phenotypic outcomes, such as cancer (Keller, 2008). Those mutations that occur in sperm or egg cells are more evolutionarily salient because they can be transferred to the fertilized ovum and, eventually, to every cell in the offspring's body, which includes the offspring's own germline cells (Keller, 2008). Common processes that lead to the emergence of mutations include environmental insults associated with exposure to mutagenic chemicals and ionizing radiation, which disrupt the normal processes of DNA replication. Advanced paternal (and to a lesser extent maternal) age is a major factor associated with the presence of de novo mutations in the germ cells of offspring (Moorjani, Gao, \& Przeworski, 2016). This stems from the age-related breakdown of mechanisms that ensure the fidelity of DNA replication during the process of meiosis (i.e., the form of genetically reductive cell division that generates haploid gametes) (Moorjani et al., 2016).

Many mutations are likely to be neutral with respect to fitness; some are deleterious, while beneficial mutations are the rarest (Arslan \& Penke, 2015). Calling a mutation "neutral" also depends on its commonness, not just its effect size, because the "neutrality" of a mutation can be reduced to chance (or drift) being more important for its fate than selection (Arslan \& 
Penke, 2015). As chance events can eliminate all copies of a mutation, a rare mutation with a small beneficial effect will have its fate determined mostly by chance. The mutation will be governed more by selection when it becomes more frequent: in larger populations with more copies of the mutation, chance becomes less important to the prevalence of a mutation (Arslan \& Penke, 2015). Another significant point is that the effect of a mutation is contextually dependent upon local environmental pressures. A mutation that disrupts an organism's circadian rhythm (for example) in such a way that favors a longer circadian period (i.e., 27 hours instead of 24) would hypothetically confer an advantage upon such a mutant were it the case that the Earth's rotation were to slow (for demonstrations of this in competition experiments involving different circadian mutant lines of Arabidopsis thaliana under artificial environments, see Woodley of Menie, Pawlik, Webb, Bruce, \& Devlin, 2019).

\section{Behavioral genetics methods}

A variety of methods exist for determining the contributions to trait variance from genetic and environmental effects. The most basic of these make qualitative determinations concerning trait heritability based on the prevalence of phenotypes within families, and in their transmissibility across generations. Sir Francis Galton was the first to consider the utility of pedigree analysis in determining the persistence of extraordinary accomplishment among family lines. In Hereditary Genius, Galton (1869) examined the pedigrees of a number of prominent Victorian families, including his own, finding considerable evidence of status persistence across generations coupled with streaks of extraordinary talent that appeared to run in families, in some cases across multiple generations.

\section{An historical aside}

An important debate, which arose at the beginning of the $20^{\text {th }}$ century, occurred between those adhering to the biometric or ancestrian school, who, following Galton, noted that offspring tend to regress to the parental trait means, coming to resemble the average of their parents in their own trait means. This suggested that heredity involved a blending of character determinants from each parent (Froggatt \& Nevin, 1971), and at the time posed a serious challenge to Darwinism, as it was not known how this "blending heredity" could lead to the cross-generational maintenance of the sorts of new variation required for selection to work (it would just blend away with each generation). Darwin recognized this shortcoming of his own theory, coming to rely more heavily on Lamarckian mechanisms (such as pangenesis, a hypothetical mechanism for heredity involving the transmission of acquired characteristics from parent to offspring) in later editions of On the Origin of Species, to make up for this perceived flaw in his theory (cf. Winther, 2000).

On the other side of this debate were the Mendelians, whose quantitative genetic inheritance models were premised on the principles of monohybrid (involving one gene) and dihybrid (involving two genes) inheritance (Froggatt \& Nevin, 1971). Trait transmission under these models assumed a pattern of segregation involving assortment among dominant and recessive alleles in such a way that leads to distinctive distributions of phenotypes among the offspring 
of parents who might be either homozygous (i.e., dominant $\mathrm{x}$ dominant, or recessive $\mathrm{x}$ recessive) or heterozygous (i.e., dominant x recessive) for a given genetic locus. A simple example of the effects of monohybrid inheritance on the distribution of phenotypes, the expression of which are dependent upon the presence of at least one dominant allele, can be illustrated thusly: imagine a parent who exhibits the symptoms of a disease that is conferred by the possession of at least one dominant allele, and is heterozygous with respect to the dominant allele (i.e., their genotype is $D d$, where the presence of $D$ indicates that the parent both carries and expresses the disease). This parent then mates with a partner who is similarly heterozygous for the same phenotype (also $D d$ ). The offspring of that mating will exhibit the disease in a 3:1 ratio, meaning that a random offspring of that mating is three times more likely to both carry and express the disease-causing dominant allele than not. This is because after segregation among these alleles, as well as (random) recombination, there will be three offspring who express the disease (i.e., $D D, D d, D d$ ), and one who neither expresses nor carries the disease phenotype $(d d)$.

Mendelians, like proponents of biometrical models of heredity, also had difficulty squaring their own findings with Darwinism, as Mendelian segregation among independently assorting alleles, especially under conditions of random mating, would tend towards these heterozygotes and homozygotes with respect to specific loci assuming what is known as Hardy-Weinberg equilibrium within a population. Hardy-Weinberg equilibrium is described by equation 1

1) $p^{2}+2 p q+q^{2}=1$

In the equilibrium, $p^{2}$ equals the prevalence of the homozygotes of dominant allele $p, 2 p q$ equals the heterozygote frequency for dominant and recessive alleles $p$ and $q$ respectively, and $q^{2}$ equals the frequency of the recessive allele heterozygotes. The tendency towards this sort of equilibrium was believed to preclude the possibility of novel and useful allelic combinations (haplotypes) arising that might advantage an incipient species adapting to a novel environment, as linkage decay would rapidly set in, "randomizing out" the effect of the beneficial allelic pairing. Mendelians tended towards saltationistic explanations to account for evolutionary change. Saltation, from the Latin saltus, meaning jump, was a mode of evolution that involved massive and discontinuous reorganization of organismal body-plans ("macromutations"), leading to very infrequent (given the rarity of such "hopeful monsters"), but theoretically very rapid evolutionary change (Stoltzfus \& Cable, 2014).

The differences between the biometric and Mendelian schools (both of which were respectively well evidenced) were finally resolved with the neo-Darwinian Synthesis in the first half of the $20^{\text {th }}$ century (discussed briefly in the Introduction). Two major discoveries paved the way for this synthesis, including the discovery of genetic mutations (Haldane, 1937) and genetic load (Muller, 1950), which could cause genes to randomly change in ways that might lead to an organism and its progeny gaining a selective advantage relative to other organisms given changing environments, but in most cases would disadvantage organisms, contributing to fitness-reducing genetic load instead (as discussed in the previous section). The second key discovery was that for many traits, genes are discrete, but are additive in 
effect, contributing individually small, but cumulatively large amounts to trait heritability as part of a polygene. For other traits, genes with large interactive or dominant effects dominate the trait architectures. These insights became key to how behavior geneticists apportion genetic variance when considering genetic contributions to trait architecture.

\subsection{Twins and other forms of phenotypic resemblance models}

Galton (1869) was also the first to propose the idea that comparisons involving identical (monozygotic) and fraternal (dizygotic) twins might allow us to usefully infer the degree to which the influence on a given trait could be due to the action of what he termed nature (i.e., genetical factors) versus what he termed nurture (i.e., environmental factors). Twin studies using essentially "modern" correlational designs appeared in the first quarter of the $20^{\text {th }}$ century (Liew, Elsner, Spector, \& Hammond, 2005). Unlike studies that capitalize on parentoffspring or sibling resemblance, these allowed for genetic influences to be precisely determined on the basis of the known zygosity of the twin sets being compared. Monozygotic twins share $100 \%$ of their genotype whereas dizygotic twins share $50 \%$ of the few alleles varying in a local population. The simplest basis for estimating heritability using twins involves subtracting the trait correlation among the monozygotic twins from that estimated for the dizygotic twins, and then doubling the resultant difference (Falconer, 1960). This is known as the Falconer's Formula, and is depicted in equation 2.

2) $H^{2}=2 *\left(r_{\mathrm{MZ}}-r_{\mathrm{DZ}}\right)$

Therefore, if we are measuring a trait (let's say extraversion) in a set of twins, and we find that the correlation in our monozygotic twin sample is 0.70 , whereas the correlation in the dizygotic twin sample is 0.50 , then the $H^{2}$ for extraversion, based on equation 2 , can be estimated as 0.4 . This means that $40 \%$ of the variance in extraversion is due to the action of genetic factors, and the remaining $60 \%$ is due to environmental factors. An important point to keep in mind while considering the above is that the heritability here is what is known as broad-sense heritability $\left(\mathrm{Big}-H^{2}\right)$. This means that it necessarily captures all sources of genetic resemblance between twins, irrespective of whether that source of genetic variance is additive, i.e., that which results from genes with summative effects on phenotypic variance (this being narrow-sense heritability, represented with a little- $h^{2}$ ), or dominance, i.e., that which results from genes with multiplicative effects on phenotypic variance. Similarly, environmentality in this equation is also broad-sense, indiscriminately capturing all sources of dissimilarity among twins stemming from the impacts of both shared (i.e., within-family effects) and non-shared (unique, or idiosyncratic influences) environments. The Big- $E^{2}$ variance component also captures measurement error, stemming from the imperfect measurement of trait variation.

Basic behavioral genetic models, such as Falconer's Formula, can be modified to use adoption designs, where adopted siblings (theoretically) share no zygosity, relative to fullblooded ones (who share 50\%). Another way in which twins can be used to determine genetic influences on a trait is via the method of concordance. This approach is used on data where the trait of interest is distributed discretely among the population, rather than continuously 
(i.e., the presence or absence of a disease). When the frequency of concordance for a given trait is significantly higher among monozygotic twins relative to dizygotic ones, the trait can be said to be heritable (Falconer, 1960).

More sophisticated behavioral genetic methods have subsequently been developed which permit the decomposition of determinants of phenotypic variance into their additive (A), shared environment (C), and non-shared environment (E) components (known as ACE models), allowing for a more granular estimation of the impacts of different sources of environmentality. Alternatively, models can be estimated that replace $\mathrm{C}$ with the effects of genetic "Dominance" (D) (Neale \& Cardon, 1992). The term "Dominance" is somewhat of a misnomer, as this variance component capitalizes on the impact of all forms of non-additive genetic variance, including both Mendelian dominance effects (where gene-by-gene [GxG] interactions among alleles of the same locus determine the pattern of trait transmission) and epistasis, this being GxG interactions involving multiple alleles and multiple loci (Eaves, Neale, \& Maes, 1996). Both sources of non-additivity enhance the resemblance of monozygotic twins relative to dizygotic ones, leading to significantly inflated estimates of heritability (with Falconer's Formula yielding broad-sense heritabilities $>1$ in extreme cases). Typically the presence of non-additivity is inferred when the monozygotic twin correlation is $\geq$ twice the dizygotic correlation (Eaves et al., 1996). Separating dominant from epistatic non-additive influences is difficult. However, some have proposed that the action of epistasis can be inferred when the dizygotic twin correlation is zero (Eaves et al., 1996).

The wide availability of inexpensive computing power has led to the development of approaches based on newer biometric structural equation modelling, such as Cholesky decomposition, a form of path analysis that can be used to determine the relative 'goodnessof-fit' of a variety of different models testing different combinations of variance components (such as ACE, ADE, AE, DE, etc.), thus expanding the scope of what can be learned about the determinants of trait variance. Pedigrees and sibship can be used to determine familial clustering for the purposes of estimating heritability when sample sizes are large (as such approaches typically yield less accurate estimates of the relevant variance components). Furthermore, when pedigree-based approaches are combined with twins, the resultant twinpedigree designs can be used to estimate additional variance components, owing to the increased diversity of relationships that can be incorporated into such models. This includes the ability to separate $\mathrm{C}$ (specifically, shared household) effects from D (non-additivity) effects (van der Zee, Helmer, Boomsma, Dolan, \& de Geus, 2020). Using spousal resemblance, such models also permit controlling for the degree of assortative mating (van der Zee et al., 2020). Assortative mating can inflate heritability estimates as it increases the degree to which offspring resemble parents (more about this in section 4.2 below).

An important point to consider when interpreting behavioral genetic data is that such models typically assume that the trait's norm of reaction (i.e., the range of environments over which it can canalize fully; also known as reaction norm) is relatively flat-i.e., the heritability of the trait should be equal across a wide range of environments. The idea that environments 
might differentially influence the expression of genes associated with the same trait constitutes a confounding factor when considering the meaning of heritability. To estimate the effects of gene-by-environment $(\mathrm{GxE})$ interactions on trait variance, the genetic variance components can be interacted with environmental ones (yielding four potential orthogonal contrasts, additive-by-non-shared environment [AxE], additive-by-shared environment $[\mathrm{AxC}]$, dominance-by-non-shared environment [DxE], and dominance-by-shared environment $[\mathrm{DxC}])$. Most commonly, $\mathrm{GxE}$ is modelled as AxE, after hierarchically estimating the main effects of A, C, and E separately (e.g., Tucker-Drob \& Bates, 2016). Comparing twins reared apart with twins reared together also yields important insights into the role of divergent environments in inducing behavioral convergence among twins. Such models have reported that identical twins separated at birth often come to more strongly resemble one another than identical twins reared together, possibly due to the lack of a need to compete for the same familial micro-niche (Segal, 2008). Such findings also militate against shared environmentality (C) as a major cause of twin convergence, as all environmentality in these cases is non-shared (Segal, 2008). Variance partitioning approaches also make the assumption that the environmental experiences of monozygotic twins are no more alike than the environmental experiences of dizygotic twins. This is referred to as the equal environments assumption, and is supported by various studies (Barbaro \& Penke, 2020).

An interesting and recent twist on twin-based approaches to estimating heritability is the approach developed by Nancy Segal (2013), which involves attempting to estimate heritability on the basis of comparing unrelated lookalikes (pseudo-twins) - these being randomly selected individuals who happen to strongly resemble one another, but have no (known) relatedness. This approach was designed to test criticisms of twin-based heritability estimates based on the idea that behavioral convergence might be a function of "equivalent treatment" effects based on appearance. The finding that these pseudo-twins exhibit limited convergence with respect to personality traits falsifies this hypothesis (Segal, 2013).

In animal models, it is often not possible to use identical and non-identical twins in the estimation of heritability. Therefore, pedigree analysis and genetic techniques constitute the standard method for estimating heritability. One of the most commonly used approaches is SOLAR (Sequential Oligogenic Linkage Analysis Routines) which uses both pedigree-based and empirically calculated kinship in the estimation of phenotypic heritability (e.g., Hopkins, Russell, \& Schaeffer, 2014).

Finally, a "classical" phenotypic approach to determining the prospective impact of mutationload on trait variance involves assessing the impact of advanced paternal age on the trait levels of offspring. This is based on the premise that older fathers, all else equal, bequeath a larger number of de novo (typically substitution-type) point mutations to offspring, which should reduce offspring trait means (all else being equal, and under the assumption that these mutations are mildly negative) (e.g., D'Onofrio et al., 2014). Molecular studies have demonstrated robust effects of paternal (and to a lesser extent maternal) age effects on the 
burdens of mutations in the genomes of offspring, with each year of advanced paternal age adding approximately 1.4 new mutations to the genomes of offspring (Moorjani et al., 2016). Phenotypic paternal age effect studies are sensitive to a variety of confounds, however, including inadequate measurement of the relevant phenotypic parameter in parents (a necessary confound in instances where it might affect age at paternity), and life history characteristics that are likely to either influence gamete quality independently of, or even counteract, the effects of parental age (Barbaro et al., 2018), and are seldom controlled in such studies.

\subsection{Molecular genetic approaches}

A variety of approaches exist for ascertaining the direct contribution to trait variance stemming from genetic factors. Among the oldest of these are candidate gene studies. These are based on the hypothesized action of a specific genetic variant on a specific phenotype, based on the former's involvement in some molecular process or expression with respect to a prospectively relevant phenotype. Candidate gene studies, at least among human subjects, have typically failed to identify variants with stable replicable effects on trait variance (for a review of the history and failure of this approach in intelligence research, see Chabris et al., 2012).

A more successful approach is polygenic scoring. This involves the use of GWAS (Genome Wide Association Studies) in which the frequencies of specific variants (SNPs) are regressed against a target phenotype (such as educational attainment). After correction for population stratification, cryptic relatedness, and false discovery, the remaining pool of variants can be summed and weighted by their individual effect sizes. The resultant weighted composite will take the form of a normally distributed variable known as a polygenic score (PGS) which can be used in standard regression analyses, as if it were any other continuously distributed predictor. Polygenic scores have the quality of removing ambiguity in effect direction, as they operationalize directly an aspect of the genetic architecture that accounts for trait variance. For some well-studied phenotypes, such as intelligence and educational attainment, PGSs can account for modest portions of trait variance. For example, one PGS estimated in Lee et al. (2018), called Educational Attainment 3 [EA3], was found to account for approximately $10 \%$ of the variance in performance on the Henmon-Nelson IQ test in the Wisconsin Longitudinal Study, as part of an out-of-sample replication. With improvements in the techniques used to identify relevant variants and also to regress variant-frequencies against target phenotypes have come increases in power to construct PGSs that account for greater portions of a trait's variance (for example, compare Rietveld et al., 2013 with Lee et al., 2018 in the case of educational attainment). As with conventional estimates of additive genetic variance in twin studies, various methods have been developed for the purpose of detecting GxE interactions on the expressivity of PGSs on their target phenotypes (e.g., Woodley of Menie, Pallesen, \& Sarraf, 2018; Woodley of Menie, Peñaherrera-Aguirre, Dunkel, \& Sarraf, 2021). 
One of the most significant developments in $21^{\text {st }}$ century behavioral genetics was a technique for estimating the heritability of a trait based on the results of genomic data alone. Known as GCTA (Genome-Wide Complex Trait Analysis), this technique uses the contributions of all SNPs on a specific chromosome, or for the entirety of the genome, to estimate "SNPheritability," the proportion of trait variance that can be accounted for by the cumulative impacts of all (sampled) SNPs. This technique capitalizes on the fact that, within a large enough sample, there will be individuals who are "genetically similar" to one another (after controls for cryptic relatedness and population stratification) by chance. This opportunistic similarity can be exploited to estimate SNP-heritabilities, as if one were comparing twins (Yang, Lee, Goddard, \& Visscher, 2011). Estimates of SNP-heritability for e.g., intelligence and educational attainment, based on the results of GCTA, are typically lower than those obtained using more conventional behavioral genetic estimates (i.e., the heritability of IQ based on conventional methods is as high as 0.8 among adults, but is substantially lower [closer to 0.4] when estimated using GCTA). This has been termed "missing GCTA heritability" (Plomin \& Deary, 2015), and might result from a variety of factors, such as partial genomic coverage, the influence of rarer variants, and non-additivity effects stemming from $\mathrm{GxG}$ interactions.

A variety of molecular techniques exist for identifying rare variants, and determining their impact on trait genetic architecture. Rare variants (minor allele frequency <0.01) are typically newly occurring mutations with deleterious effects on the phenotype. These can take the form of point mutations, especially those occurring in the exome (the region of the genome responsible for protein coding), or copy number variations (CNVs), which involve the duplication, deletion, or inversion of segments of DNA. Genome-wide studies of CNV counts have found little evidence for an effect of these on traits such as intelligence (Bagshaw et al., 2013). However, more recently, familial studies have found a substantial role for rare variants in the maintenance of variance in intelligence and educational attainment. Specifically, one recent study (Hill et al., 2018) employed 20,000 individuals in the Generation Scotland family cohort genotyped for $\sim 700,000$ SNPs. This allowed the use of high levels of withinfamily linkage disequilibrium to estimate genetic effects on intelligence, educational attainment, and personality stemming from genetic variants that are not tagged in GWASs involving unrelated individuals. Using this approach, approximately $50 \%$ of the variance in intelligence, and approximately $40 \%$ of the variance in education could be accounted for when large numbers of prospectively rare variants were included in the estimate of SNPheritability. Hill et al. (2018) concluded that the prominence of rare variants in the genetic architecture of these cognitive phenotypes is strongly suggestive of the operation of mutationselection balance on these traits.

Finally, it needs to be noted that recently, attention is being paid to the role of what are termed indirect genetic effects (IGE) on the canalization of phenotypic variance within human populations (although these effects are not restricted to humans; Linksvayer, 2007). One class of IGE in particular, termed social epistasis, has been the subject of recent research (Domingue \& Belsky, 2017; Woodley of Menie, Kanazawa, Pallesen, \& Sarraf, 2020). Social epistatic effects stem from the ability of the aggregate frequencies of variants present within 
an individual's social genome (this being the aggregate of genomes that make up the population within which an individual is embedded) to influence the expressivity of an individual's own genetic variants for a given trait. Domingue et al. (2018) demonstrated the action of social epistasis effects as a moderator of the expressivity of genetic variants associated with a PGS for educational attainment in a large, representative genotyped sample of the US population. They found that the average PGS of classmates positively moderated the degree to which PGS predicted phenotypic variance in educational attainment. That is to say, in schools where the average PGS level among students was higher, individual PGSs were better able to express the relevant phenotypes that contribute to educational attainment. IGEs confound heritability estimates, as they constitute genetic influences on canalization that stem from outside of the individual genome. In mice, Baud et al. (2017) found that up to $29 \%$ of the apparently heritable variance in anxiety and immune functioning among mice was due to the influence of horizontal IGEs. Recent studies on human populations have found indications of substantial influences on trait variance stemming from horizontal IGEs (Xia, Canela-Xandri, Rawlik, \& Tenesa, 2020).

Recognition that much of what is generically termed "environmentality" among human populations is in fact indirect genetic variance epistatically influencing individual patterns of gene expression will allow for better models to be developed in the study of so-called GxE interactions.

\section{Showcasing behavioral genetics in sex research with case studies}

In this section, we discuss a few landmark behavioral and molecular genetic studies, focussing on the field of sex research to situate our examples within the scope of this Handbook.

\subsection{GWAS: Same-sex sexual behavior}

Although prenatal hormonal events exert substantial influence on sexual orientation in humans and non-human animals (Luoto et al., 2019a; Luoto, Krams, \& Rantala, 2019b; Swift-Gallant, 2019), genetic factors also play a role in inducing variation in human sexual orientation and behavior. Additionally, there are various ways in which genetic factors can interact with prenatal developmental events, causing variation in a range of phenotypes from morphometrics, cognition, behavioral dispositions, and sexual orientation (Luoto et al., 2019a, 2019b; Rahman \& Wilson, 2003). This variation, and the evolutionary-developmental mechanisms underlying it, is described in more detail in the chapter by Luoto and Rantala (2021) in this Handbook and we refer interested readers to that chapter for a more detailed elaboration. 
A significant and recent genome-wide association study (GWAS) sought to identify genes associated with same-sex sexual preferences in humans (Ganna et al., 2019). The study in question, published in Science in 2019, was not first to explore the genetic factors underlying human sexual orientation, but it is based on an unusually large sample of 477,552 individuals from the UK and the US, and unlike prior genetic studies on human sexual orientation, it meets current standards of genome-wide statistical significance $\left(P<5 \times 10^{-8}\right)$ (Ganna et al., 2019). The study focussed only on participants of European ancestry to prevent betweenpopulation differences from obfuscating the results. This kind of population-specificity is preferable in large-scale GWASs for obtaining clearer results.

The results revealed five autosomal loci associated with same-sex sexual behavior in men and women (Ganna et al., 2019). The loci suggested links to biological pathways that involve sex hormone regulation and olfaction. Two genome-wide significant signals for same-sex sexual behavior (rs11114975-12q21.31 and rs10261857-7q31.2) were shared between men and women. Sex-specific analyses showed two additional signals for same-sex sexual behavior in men (rs28371400-15q21.3 and rs34730029-11q12.1) which showed no significant association in women, and one in women (rs13135637-4p14) which showed no significant association in men (Ganna et al., 2019). The genetic influences on same-sex sexual behavior were positively but imperfectly correlated between the sexes, the genetic correlation coefficient being $r_{\mathrm{g}}=0.63$ (Ganna et al., 2019). This suggests that the genetic basis of same-sex sexual behavior is moderately sex-specific: most other traits show higher genetic correlations between the sexes, often close to 1 (Ganna et al., 2019). In a meta-analysis of three independent replication samples ( $n=2308$ US adult males; $n=4755$ US young adults; $n=$ 8093 Swedish adolescents), three of the loci underlying same-sex sexual behavior remained significantly correlated with different but related dependent variables, namely sexual identity and same-sex attraction, as did a polygenic score of same-sex sexual behavior constructed on the basis of the larger discovery sample (Ganna et al., 2019). Overall, the analyses reported by Ganna and colleagues (2019) showed that many loci underlie same-sex sexual behavior in both sexes, although only a few loci passed the stringent statistical corrections for genomewide multiple testing and were replicated in other samples.

Taken together, all the tested genetic variants accounted for $8-25 \%$ of the variation in male and female same-sex sexual behavior (Ganna et al., 2019). This SNP-based heritability is lower than the $32 \%$ broad-sense heritability (i.e., the percentage of variation in a trait attributable to all sources of genetic variation) reported in family-based methods; as such, it is consistent with empirical findings for other traits in which family heritability is approximately three times larger than SNP-heritability (Ganna et al., 2019). The polygenic scores based on the discovery sample were also associated with sexual attraction in participants at age 15 years $\left(p=6.4 \times 10^{-5}\right)$ in a replication sample, which suggests that at least some of the genetic influences on same-sex sexual behavior manifest also in same-sex sexual attraction early in sexual development (Ganna et al., 2019). Nevertheless, the variance explained by the polygenic scores was extremely low $(<1 \%)$ in all cases, meaning that the polygenic scores could not be used to accurately predict sexual behavior at an individual level (Ganna et al., 2019). 
Those nonheterosexual individuals (both men and women) with a greater proportion of samesex partners had a larger reproductive disadvantage (lower birth-year adjusted number of children) relative to exclusively heterosexual participants (Ganna et al., 2019). However, this fitness impairment may be offset by the reported higher number of offspring in nonheterosexual women's kin compared with the kin of heterosexual women (Camperio Ciani, Battaglia, Cesare, Camperio Ciani, \& Capiluppi, 2018), indicating sexually antagonistic selection (Luoto et al., 2019a). Sexually antagonistic selection refers to the fact that the attributes favored in one sex are sometimes opposed to those favored in the other sex, which may lead to a fitness detriment when the genotype results in offspring of one sex and a fitness increase when it results in offspring of the other sex (Luoto et al., 2019a). The kin selection hypothesis, which posits that homosexuality is retained in populations because homosexual individuals either directly or indirectly enhance the fitness of heterosexual siblings, is generally not supported in homosexual men in Western countries and Japan, though some supporting evidence has been reported in Samoa and Indonesia (Nila, Barthes, Crochet, Suryobroto, \& Raymond, 2018). However, kin selection alone is considered insufficient for explaining the maintenance of male homosexuality (Nila et al., 2018), and other hypotheses are therefore required for understanding the prevalence of same-sex sexual behavior and attraction across species, countries, and historical time (see chapters XX-XX, this volume).

\subsection{GWAS: Assortative mating}

Another landmark GWAS in genetically informed sex research was published in the inaugural volume of Nature Human Behaviour in 2017. Robinson and colleagues (Robinson et al., 2017) studied assortative mating (see Glossary in Table 1), focussing on both phenotypic and genetic evidence of this mating pattern. That is, the study sought to determine the degree to which individuals with similar phenotypes and/or similar genotypes mate with one another more frequently than expected by chance. Assortative mating is evolutionarily relevant for several reasons. For instance, when there is direct phenotypic selection for a heritable trait, reproduction between phenotypically and genotypically similar individuals will increase the proportion of homozygous offspring, creating a directional build-up of linkage disequilibria after many generations of selection. This in turn will increase trait correlations between relatives, and induce indirect assortative mating on other traits that are genetically correlated but not directly selected for in mate choice (Robinson et al., 2017; see also Conroy-Beam et al., 2019).

Although it has been known for more than a century that phenotypically similar individuals mate assortatively with respect to traits such as intelligence and height, the genetic underpinnings and/or consequences of this phenomenon are less well understood (Robinson et al., 2017). The main phenotypic traits analyzed by Robinson et al. (2017) were height and BMI, using three independent samples of a total of 24,662 spousal pairs of European ethnicity. The study also analyzed assortative mating for waist-to-hip ratio, systolic blood 
pressure, and educational attainment using another sample of 7,780 UK-based couples (Robinson et al., 2017). All couples were genotyped, and all couples had a child together (inferred from genotype data and/or known pedigree structure).

Robinson and colleagues (2017) began by estimating the phenotypic correlation among couples for height and BMI, controlling for age and sex differences in both traits. Next, the authors predicted an individual's phenotype from a genome-wide genetic predictor created from their partner's genotype. This step was followed by a re-estimation of the SNP effects on the target phenotypes in a random-effect model that converts the least-squares SNP estimates into so-called "approximate best linear unbiased predictors" (that is, summary statistic BLUP: SBLUP). By creating a genetic predictor with BLUP properties, the SBLUP method maximizes predictive power. The SBLUP predictors for height and BMI found by Robinson et al. (2017) explained $18 \%$ of the phenotypic variation of height and $8 \%$ of the phenotypic variation of BMI, which was similar to the estimates of $17 \%$ and $7 \%$, respectively, that were obtained by using genetic predictors made directly from GWAS summary statistics.

The authors used a mixed-effects model to estimate the regression coefficient from a linear regression of the phenotype of one partner on the SBLUP genetic predictor of the other partner, and vice versa. Theory and simulations indicated that if there is direct assortative mating for a phenotype, and the predictor has "BLUP properties" (i.e., slope of the regression of phenotype on genetic predictor of $\sim 1$ ), the regression coefficient from a linear regression of the phenotype of one partner on the genetic predictor of the other partner should equal the phenotypic correlation among couples (Robinson et al., 2017). According to theory and simulations, indirect assortment for an unmeasured genetically correlated trait would also result in a correlation among couples at trait-associated loci for the recorded phenotype, with the value of the correlation being dependent on the phenotypic and genetic correlations of the different phenotypes, the ratio of their heritability, and the degree of partner assortment (Robinson et al., 2017). The methods used by Robinson and colleagues therefore provided a direct estimate of the correlation among couples at trait-associated loci, but without differentiating between direct assortment on a phenotype and assortment on a genetically correlated trait. What this method does differentiate is assortative mating based on selection of phenotypic characteristics and assortative mating based on shared social or environmental factors: if there was only social/environmental assortative mating, an association between genetic predictors of phenotype would not emerge (Robinson et al., 2017).

Using these methods, Robinson and colleagues (2017) found evidence of a genetic basis for assortative mating on both height and BMI. Across all of their samples, the meta-analyzed phenotypic correlation among partners was 0.201 for height and 0.228 for BMI. Furthermore, the meta-analyzed value of the regression coefficient from a linear regression of the SBLUP genetic predictor of males and the height phenotype of their female partner, and vice versa, was similar (meta-analyzed value $=0.200$ ) to the phenotypic correlation. The meta-analyzed estimate of the regression coefficient for BMI was 0.143, which was lower than the 
phenotypic correlation. The regression coefficients for both height and BMI were different from the predicted value of zero which would be expected if there was only social (nongenetic) assortative mating (Robinson et al., 2017). The results suggest that there is direct genetic and phenotypic assortative mating on height. If these patterns were indirectly driven by assortative mating on another related trait, obtaining a genetic estimate equal to the phenotypic estimate under indirect assortment would require a combination of (1) a partner correlation that is greater than 0.2 on the said traits, (2) that trait having a genetic correlation of $>0.5$ with height, and (3) heritability of $>0.8$. It is unlikely that any trait meets these criteria, which therefore indicates direct genetic and phenotypic assortative mating on height (Robinson et al., 2017). Robinson and colleagues' results for BMI suggested a few alternatives. There may be indirect assortative mating on a genetically correlated trait, or there may be direct assortative mating on BMI combined with environmental factors that result in phenotypic convergence among partners (Robinson et al., 2017). Whatever the mechanism, the results provided evidence of assortative mating at genetic loci associated with height and BMI, which implies linkage disequilibrium (that is, the non-random association of alleles at different loci in a given population) at those loci (Robinson et al., 2017).

The authors further reported a correlation among partners at trait-associated loci for WHR, blood pressure, and educational attainment, but no evidence for either a phenotypic or a genotypic correlation for bone mineral density. For educational attainment, the genetic correlation at trait-associated loci (0.654) was significantly higher than the phenotypic correlation (0.412) (Robinson et al., 2017), which suggests the existence of selection on a trait or traits genetically correlated with educational attainment.

\subsection{Other behavioral and molecular genetics studies in evolutionary sex research}

There are many more studies that we could have highlighted in this section through in-depth discussions to provide readers with a broader introduction of behavioral, and in particular the latest molecular genetics research in evolutionary sex research. To save the reader the trouble of going through all of that detail, we here provide a brief synopsis of a few recent studies, which helps us broaden the scope of this section without laboring on the details of each study. Readers wanting to know more about genetic assortative mating in humans may, for instance, be interested in a recent large-scale study by Nishi and colleagues (2020), which reported that the group of SNPs exhibiting the highest assortative mating had higher signals of recent positive selection. Therefore, assortative mating might affect changes in allele frequency over time, ultimately accelerating the frequency of advantageous alleles (Nishi et al., 2020).

Besides the GWAS approach to same-sex sexual behavior discussed in section 4.1, there have also been many twin studies seeking to disentangle the extent to which genetic vs. environmental factors co-contribute to same-sex sexual attraction and/or behavior (see section 3.1 for an explanation of how twin studies are used in behavioral genetics). A review of such studies concluded that the heritability of nonheterosexual orientation is 0.32 , 
indicating that approximately a third of the between-individual variation in sexual orientation can be attributed broadly to genetic differences between individuals (Bailey et al., 2016). This review pooled twin studies on male and female nonheterosexuality without distinguishing between them; however, there is substantial evidence indicating that the proximate mechanisms underlying male and female nonheterosexuality are different (Luoto, 2020; Luoto et al., 2019b; Swift-Gallant, Coome, Aitken, Ashley Monks, \& VanderLaan, 2019). For this reason, it is necessary to analyze nonheterosexuality with a higher degree of granularity, focussing separately on the ultimate functions and proximate mechanisms underlying male and female sexual orientation, including different types of nonheterosexual individuals in men and women (e.g., masculine "butches" and feminine "femmes") (Luoto et al., 2019a, 2019b; Swift-Gallant et al., 2019).

In another line of research moored in evolutionary life history theory, Minkov and Bond (2015) conducted a cross-national sociogenetic study on polymorphisms in three life history candidate genetic loci- the androgen receptor gene (AR), the dopamine-receptor gene (DRD4), and the serotonin transporter gene (SLC6A4). Minkov and Bond compared the aggregate life-history strategy genetic factor arising from these genetic loci with indicators of national-level life history strategies. The authors found that the aggregate life history strategy genetic factor which was theoretically predicted to be correlated with faster life history strategies at the individual level also did so at the national level. As the three genetic indices formed a strong single factor at the national level, it is possible that they had a synergistic effect at the population level which reflected the synergistic effect of dopamine and serotonin at the individual level (Minkov \& Bond, 2015). The observation that the life history strategy genetic factor arising from this study was also strongly correlated with a proxy of nationallevel life history strategy and time orientation (Minkov \& Bond, 2015) suggests that genetic differences between populations may contribute to broader differences in outcome variables related to life history strategies. Similar results were reported by another study which found a high correlation between national-level adolescent fertility rates (used as a population-level life history marker) and Minkov and Bond's life history strategy genetic factor index, further suggesting the existence of genetic factors underlying population-level life history variation (Luoto, 2019b). For a recent discussion on the heritability of life history strategies at the level of individuals, see Woodley of Menie et al. (2021).

Although single genes produce limited differences in personality, cognition, and behavior at the individual level, national-level analyses often produce more significant associations between polymorphisms in some genetic loci and relevant national-level outcome variables (Minkov \& Bond, 2015). A possible explanation for this discrepancy between the individual level and the national level is that the national-level variation in certain genetic loci may be driven by a significantly larger number of genes, each of which produces a very small or no effect at the individual level but a large cumulative effect at the level of nations (Minkov \& Bond, 2015). Thus, it is possible that genetic factors comprising only a few key genetic loci (such as Minkov and Bond's life history strategy genetic factor) act as a proxy for a much larger collection of alleles across various genetic loci which may or may not be in linkage disequilibrium at the level of individuals (that is, they may or may not co-occur in the same 
individuals). However such alleles may be linked at the level of individuals, the frequencies of these genes can correlate at the national level, producing an effect large enough to be discernible in cross-national analyses of only a few key genetic loci and relevant outcome variables (Minkov \& Bond, 2015).

Evolutionarily informed sex researchers have gained substantial insights into the study of human behavior through comparative analyses in non-human animals, which also enable many experimental protocols that would be unethical in humans. Owing to the fact that most of the neurobiological mechanisms governing human cognition and behavior are evolutionarily conserved, or at least show significant phylogenetic inertia (Luoto \& Varella, 2021; Panksepp, 1998), the fields of comparative physiology, genomics, cognition, and behavior can offer many opportunities for a more consilient understanding of the evolutionary underpinnings of human cognition and behavior than is possible with analytical approaches focussing only on a single species. Some of the insight gleaned from this type of comparative research includes the identification of polymorphisms in genetic loci that account for variance in behavioral phenotypes across species. For instance, research on voles and humans has identified shared neurobiological mechanisms underlying pair-bonding in both species. The neuropeptides oxytocin and vasopressin and the neurotransmitter dopamine facilitate pair-bonding, while polymorphisms in oxytocin, vasopressin, and dopamine genes, and their receptor-rich brain regions, are involved in basic motivational processes, sexual satisfaction, and pair-bonding across species (Acevedo, Poulin, Collins, \& Brown, 2020; Diekhof, Richter, Brodmann, \& Gruber, 2021). These kinds of findings provide evidence that pair-bonding and romantic love maintenance in humans are parts of a broad mammalian strategy for reproduction and long-term attachment which is influenced by basic reward circuitry and genetic factors that create individual differences in sexual behaviors, sexual preferences, and pair-bonding (Acevedo et al., 2020; Bode \& Kushnick, 2021; Luoto et al., 2019a). For more detailed discussions of these topics, readers may wish to refer to Acevedo et al. (2020) who used a mixed methods approach — both neuroimaging and gene sampling to study how genetic polymorphisms and brain activation are related to romantic love in newlywed couples (see also Bode \& Kushnick, 2021, for a broader review). Another study by Diekhof and colleagues (2021) genotyped participants for genetic polymorphisms in the dopamine system and created a multilocus genetic composite (MGC) score which reflected the additive effect of different alleles conferring relative increased dopamine transmissionwhich in turn influences reward processes and behaviors across species (see also Kringelbach \& Berridge, 2010; Luoto et al., 2019b; Struik, Sanna, \& Fattore, 2018). Finally, we wish to highlight a recent study by Pearce and colleagues (2019) which found that individual differences in dopamine gene polymorphisms indirectly influenced men's sociosexuality through increased impulsivity. In women, individual differences in endorphin and vasopressin gene polymorphisms were associated with openness to experience, which mediated the indirect effects of these polymorphisms on sociosexuality (Pearce et al., 2019).

In the aggregate, studies like these provide important details on how individual, population, and even species differences in genomes ultimately underlie variability in behavioral dispositions, motivational priorities, and even life outcomes. By way of summary, we 
conclude with reporting the ten most robustly replicated findings from behavioral genetics (Table 2).

Table 2. The top ten replicated findings from behavioral genetics. Adapted from Plomin et al. (2016).

Finding 1. All psychological traits show significant and substantial genetic influence.

Finding 2. No traits are 100\% heritable. (Though individual differences in some traits, such as height and executive functioning, yield heritabilities $>90 \%$, and as high as $99 \%$ in the case of executive functioning)

Finding 3. Heritability is caused by many genes of small effect.

Finding 4. Phenotypic correlations between psychological traits show significant and substantial genetic mediation. Such correlations, therefore, do not arise from simple environmentally driven covariance but also have a genetic covariance underlying them.

Finding 5. The heritability of intelligence increases throughout development (this is called the Wilson Effect). This is an interesting finding because other domains such as personality do not show systematic changes in heritability during development.

Finding 6. Age-to-age stability is mainly due to genetics. Phenotypic correlations from age to age are largely due to genetic stability. That is to say, genetic effects contribute to continuity (the same genes affect the trait across age), whereas age-to-age change primarily arises because of environmental factors.

Finding 7. Most measures of the "environment" show significant genetic influence. This is because our environments are in part shaped by the genotypes and phenotypes of those around us (indirect genetic effects).

Finding 8. Most associations between environmental measures and psychological traits are significantly mediated genetically. This suggests that instead of a passive model of imposed environments influencing how genotypes develop into phenotypes, we should think about active models of shaped experiences in which humans select, modify, and create lifestyles and experiences in part based on their genetic propensities.

Finding 9. Most environmental effects are not shared by children growing up in the same family. This basic and frequently replicated finding remains one of the most far-reaching findings from behavioral genetics.

Finding 10. Abnormal is normal. The normal distribution of polygenic scores suggests that what are referred to as "disorders" are the quantitative extremes of the same genetic factors that affect the rest of the distribution, and are therefore not qualitatively distinct from the "normal" range of phenotypes (this being the cosmobian thesis). 


\section{Conclusion}

The reader of this chapter will hopefully have now attained a clearer understanding of the rationale and methods for using behavioral and molecular genetics approaches in evolutionary behavioral science and evolutionary psychology. Evolution at its most basic level refers to the change in the frequencies of alleles in a population which arises as a result of different forms of selection acting on heritable differences influencing the survival and reproduction of individuals that carry those alleles - as well as the "random" processes of genetic drift, mutation, and migration. For this reason, evolutionary behavioral scientists who work on theoretical models and empirical paradigms need to consider the multiple ways in which evolution has shaped organisms, their genomes, and their behavioral dispositions in the diverse ways that exist in nature - including in contemporary human populations. These processes are ultimately responsible for the substantial psychobehavioral variation and its heritable origins that have been noted within and between different human populations. Paying greater attention to the genetic underpinnings of human behavior and developmental psychobiology may eventually yield an evolutionary psychological research paradigm that is scientifically more robust, explanatorily powerful, and consilient with recent developments in evolutionary biology. We hope that this overview of relevant behavioral genetics methods and findings, coupled with an understanding of the overarching theoretical background within which evolutionary scientists operate, provides a platform for further learning as well as inspiration for ideas concerning new and innovative research avenues under the broad aegis of what could be termed evolutionary behavioral genetics. 


\section{Acknowledgements}

The authors wish to thank Marco Antônio Corrêa Varella and Todd Shackelford for their comments on this chapter.

References

Acevedo, B. P., Poulin, M. J., Collins, N. L., \& Brown, L. L. (2020). After the Honeymoon: Neural and Genetic Correlates of Romantic Love in Newlywed Marriages. Frontiers in Psychology, 11, 634. https://doi.org/10.3389/fpsyg.2020.00634

Arslan, R. C., \& Penke, L. (2015). Evolutionary Genetics. In D. M. Buss (Ed.), The Handbook of Evolutionary Psychology (pp. 1047-1066). https://doi.org/10.1002/9781119125563.evpsych245

Bagshaw, A. T. M., Horwood, L. J., Liu, Y., Fergusson, D. M., Sullivan, P. F., \& Kennedy, M. A. (2013). No Effect of Genome-Wide Copy Number Variation on Measures of Intelligence in a New Zealand Birth Cohort. PLoS ONE, 8, e55208. https://doi.org/10.1371/journal.pone.0055208

Bailey, J. M., Vasey, P. L., Diamond, L. M., Breedlove, S. M., Vilain, E., \& Epprecht, M. (2016). Sexual orientation, controversy, and science. Psychological Science in the Public Interest, 17(2), 45- 101. https://doi.org/10.1177/1529100616637616

Barbaro, N., \& Penke, L. (2020). Behavior genetics. In SAGE Handbook of Evolutionary Psychology, Vol. 1 (pp. 336 - 354). London: SAGE.

Barbaro, N., Shackelford, T. K., Holub, A. M., Jeffery, A. J., Lopes, G. S., \& Zeigler-Hill, V. (2018). Life history correlates of human (Homo sapiens) ejaculate quality. Journal of Comparative Psychology, 133, 294-300. https://doi.org/10.1037/com0000161

Barkow, J., Cosmides, L., \& Tooby, J. (1992). The adapted mind: Evolutionary psychology and the generation of culture. New York, NY: Oxford University Press.

Bateson, P., \& Gluckman, P. (2011). Plasticity, robustness, development and evolution. Cambridge: Cambridge University Press.

Baud, A., Mulligan, M. K., Casale, F. P., Ingels, J. F., Bohl, C. J., Callebert, J., ... Stegle, O. (2017). Genetic variation in the social environment contributes to health and disease. PLoS Genetics, 13, e1006498. https://doi.org/10.1371/journal.pgen.1006498

Bode, A., \& Kushnick, G. (2021). Proximate and Ultimate Perspectives on Romantic Love. Frontiers in Psychology, 12, 573123. https://doi.org/10.3389/fpsyg.2021.573123

Camperio Ciani, A., Battaglia, U., Cesare, L., Camperio Ciani, G., \& Capiluppi, C. (2018). Possible balancing selection in human female homosexuality. Human Nature, 29(1), 14 32. https://doi.org/10.1007/s12110-017-9309-8

Chabris, C. F., Hebert, B. M., Benjamin, D. J., Beauchamp, J., Cesarini, D., van der Loos, M., ... Laibson, D. (2012). Most reported genetic associations with general intelligence are probably false positives. Psychological Science, 23, 1314-1323. 
https://doi.org/10.1177/0956797611435528

Conroy-Beam, D., Roney, J. R., Lukaszewski, A. W., Buss, D. M., Asao, K., Sorokowska, A., ... Zupančič, M. (2019). Assortative mating and the evolution of desirability covariation. Evolution and Human Behavior, 40(5), 479-491. https://doi.org/10.1016/j.evolhumbehav.2019.06.003

Cornwallis, C. K., \& Uller, T. (2010). Towards an evolutionary ecology of sexual traits. Trends in Ecology \& Evolution, 25(3), 145-152.

Crespi, B. (2020). Evolutionary and genetic insights for clinical psychology. Clinical Psychology Review. Retrieved from https://doi.org/10.1016/j.cpr.2020.101857

D’Onofrio, B. M., Rickert, M. E., Frans, E., Kuja-Halkola, R., Almqvist, C., Sjölander, A., ... Lichtenstein, P. (2014). Paternal age at childbearing and offspring psychiatric and academic morbidity. JAMA Psychiatry, 71, 432-438. https://doi.org/10.1001/jamapsychiatry.2013.4525

Daniele, V. (2021). Socioeconomic inequality and regional disparities in educational achievement: The role of relative poverty. Intelligence, 84, 101515.

Del Giudice, M. (2020). Rethinking the fast-slow continuum of individual differences. Evolution and Human Behavior, 41, 536-549. https://doi.org/10.1016/j.evolhumbehav.2020.05.004

Diekhof, E. K., Richter, A., Brodmann, K., \& Gruber, O. (2021). Dopamine multilocus genetic profiles predict sex differences in reactivity of the human reward system. Brain Structure and Function. https://doi.org/10.1007/s00429-021-02227-6

Domingue, B. W., \& Belsky, D. W. (2017). The social genome: Current findings and implications for the study of human genetics. PLoS Genetics, 13, e1006615. https://doi.org/10.1371/journal.pgen.1006615

Domingue, B. W., Belsky, D. W., Fletcher, J. M., Conley, D., Boardman, J. D., \& Harris, K. M. (2018). The social genome of friends and schoolmates in the National Longitudinal Study of Adolescent to Adult Health. Proceedings of the National Academy of Sciences of the United States of America, 115, 702-707. https://doi.org/10.1073/pnas.1711803115

Eaves, L. J., Neale, M. C., \& Maes, H. (1996). Multivariate multipoint linkage analysis of quantitative trait loci. Behavior Genetics, 26, 519-525. https://doi.org/10.1007/BF02359757

Ellis, B. J., Abrams, L. S., Masten, A. S., Sternberg, R. J., Tottenham, N., \& Frankenhuis, W. E. (2020). Hidden talents in harsh environments. Development and Psychopathology, 119. https://doi.org/10.1017/S0954579420000887

Ellis, B. J., \& Del Giudice, M. (2018). Developmental Adaptation to Stress: An Evolutionary Perspective. Annual Review of Psychology, 70(1), 111-139. https://doi.org/10.1146/annurev-psych-122216-011732

Ellison, P. T. (2017). Endocrinology, energetics, and human life history: A synthetic model. Hormones and Behavior, 91, 97-106. https://doi.org/10.1016/j.yhbeh.2016.09.006

Falconer, D. S. (1960). Introduction to quantitative genetics. Edinburgh/London, UK: Oliver \& Boyd. 
Figueredo, A. J., Sefcek, J. A., Vasquez, G., Brumbach, B. H., King, J. E., \& Jacobs, W. J. (2005). Evolutionary personality psychology. In D. M. Buss (Ed.), Handbook of evolutionary psychology (pp. 851-877). Hoboken, NJ: Wiley.

Figueredo, A. J., Vasquez, G., Brumbach, B. H., \& Schneider, S. M. R. (2004). The heritability of life history strategy: the k-factor, covitality, and personality. Social Biology, 51(3-4), 121-143.

Fisher, R. A. (1930). The genetical theory of natural selection. Oxford, UK: Oxford University Press.

Frankenhuis, W. E., \& Panchanathan, K. (2011). Individual differences in developmental plasticity may result from stochastic sampling. Perspectives on Psychological Science, 6, 336-347. https://doi.org/10.1177/1745691611412602

Froggatt, P., \& Nevin, N. C. (1971). The "law of ancestral heredity" and the Mendelianancestrian controversy in England, 1889-1906. Journal of Medical Genetics, 8, 1-36. https://doi.org/10.1136/jmg.8.1.1

Galton, F. (1869). Hereditary genius. London, UK: Macmillan.

Gangestad, S. W. (2010). Evolutionary biology looks at behavior genetics. Personality and Individual Differences, 49, 289-295. https://doi.org/10.1016/j.paid.2010.03.005

Ganna, A., Verweij, K. J. H., Nivard, M. G., Maier, R., Wedow, R., Busch, A. S., ... Zietsch, B. P. (2019). Large-scale GWAS reveals insights into the genetic architecture of samesex sexual behavior. Science, 365(6456). https://doi.org/10.1126/science.aat7693

Gould, S. J. (2002). The structure of evolutionary theory. Cambridge, MA: Belknap Press.

Haldane, J. B. S. (1937). The Effect of Variation of Fitness. The American Naturalist, 71, 337-349. https://doi.org/10.1086/280722

Heyes, C. (2012). New thinking: The evolution of human cognition. Philosophical Transactions of the Royal Society B: Biological Sciences, 367(1599), 2091-2096. https://doi.org/10.1098/rstb.2012.0111

Hill, W. D., Arslan, R. C., Xia, C., Luciano, M., Amador, C., Navarro, P., .. Penke, L. (2018). Genomic analysis of family data reveals additional genetic effects on intelligence and personality. Molecular Psychiatry, 23(12), 2347-2362. https://doi.org/10.1038/s41380-017-0005-1

Hopkins, W. D., Russell, J. L., \& Schaeffer, J. (2014). Chimpanzee intelligence is heritable. Current Biology, 24, 1649-1652. https://doi.org/10.1016/j.cub.2014.05.076

Houle, D. (2000). Is there a g factor for fitness. In G. R. Bock, J. A. Goode, \& K. Webb (Eds.), The nature of intelligence (pp. 149 -170). Chichester, UK: Wiley Ltd.

Keller, M. C. (2008). The Role of Mutations in Human Mating. In G. Geher \& G. F. Miller (Eds.), Mating intelligence: Sex, relationships, and the mind's reproductive system (pp. 173-192). Psychology Press.

Krams, I., Luoto, S., Rubika, A., Krama, T., Elferts, D., Krams, R., ... Rantala, M. J. (2019). A head start for life history development? Family income mediates associations between height and immune response in men. American Journal of Physical Anthropology, 168(3), 421-427. https://doi.org/10.1002/ajpa.23754 
Kringelbach, M. L., \& Berridge, K. C. (2010). Pleasures of the brain. Oxford University Press, USA.

Lee, J. J., Wedow, R., Okbay, A., Kong, E., Maghzian, O., Zacher, M., ... Turley, P. (2018). Gene discovery and polygenic prediction from a genome-wide association study of educational attainment in 1.1 million individuals. Nature Genetics, 50(8), 1112-1121. https://doi.org/10.1038/s41588-018-0147-3

LeVay, S. (1994). The Sexual Brain. MIT Press.

Lewis, D. M. G., Conroy-Beam, D., Asao, K., \& Buss, D. M. (2017). Evolutionary psychology: A how-to guide. American Psychologist, 72(4), 353-373.

Liew, S. H. M., Elsner, H., Spector, T. D., \& Hammond, C. J. (2005). The first "classical" twin study? Analysis of refractive error using monozygotic and dizygotic twins published in 1922. Twin Research and Human Genetics, 3, 198-200. https://doi.org/10.1375/1832427054253158

Linksvayer, T. A. (2007). Ant species differences determined by epistasis between brood and worker genomes. PLoS ONE, 2, e994. https://doi.org/10.1371/journal.pone.0000994

Luoto, S. (2019a). An updated theoretical framework for human sexual selection: From ecology, genetics, and life history to extended phenotypes. Adaptive Human Behavior and Physiology, 5(1), 48-102. https://doi.org/10.1007/s40750-018-0103-6

Luoto, S. (2019b). Response to Commentaries: Life History Genetics, Fluid Intelligence, and Extended Phenotypes. Adaptive Human Behavior and Physiology, 5(1), 112-115. https://doi.org/10.1007/s40750-019-0109-8

Luoto, S. (2020). Did prosociality drive the evolution of homosexuality? Archives of Sexual Behavior, 49, 2239-2244. https://doi.org/10.1007/s10508-020-01749-0

Luoto, S., Krams, I., \& Rantala, M. J. (2019a). A life history approach to the female sexual orientation spectrum: Evolution, development, causal mechanisms, and health. Archives of Sexual Behavior, 48(5), 1273-1308. https://doi.org/10.1007/s10508-018-1261-0

Luoto, S., Krams, I., \& Rantala, M. J. (2019b). Response to Commentaries: Life history evolution, causal mechanisms, and female sexual orientation. Archives of Sexual Behavior, 48(5), 1335-1347. https://doi.org/10.1007/s10508-018-1261-0

Luoto, S., \& Rantala, M. J. (2021). Female bisexuality. In The Cambridge Handbook of Evolutionary Perspectives on Sexual Psychology. Cambridge University Press.

Luoto, S., \& Varella, M. A. C. (2021). Pandemic leadership: Sex differences and their evolutionary-developmental origins. Frontiers in Psychology, 12, 633862. https://doi.org/10.3389/fpsyg.2021.633862

Mahner, M., \& Kary, M. (1997). What exactly are genomes, genotypes and phenotypes? And what about phenomes? Journal of Theoretical Biology, 186, 55-63. https://doi.org/10.1006/jtbi.1996.0335

Marciniak, S., \& Perry, G. H. (2017). Harnessing ancient genomes to study the history of human adaptation. Nature Reviews Genetics, 18(11), 659-674. https://doi.org/10.1038/nrg.2017.65

Miller, G. F. (2000). Sexual selection for indicators of intelligence. In G. R. Bock, J. A. 
Goode, \& K. Webb (Eds.), The nature of intelligence (pp. 260-275). Chichester, UK: Wiley Ltd.

Minkov, M., \& Bond, M. H. (2015). Genetic polymorphisms predict national differences in life history strategy and time orientation. Personality and Individual Differences, 76, 204-215.

Moorjani, P., Gao, Z., \& Przeworski, M. (2016). Human germline mutation and the erratic evolutionary clock. PLoS Biology, 14, e2000744. https://doi.org/10.1371/journal.pbio.2000744

Muller, H. J. (1950). Our load of mutations. American Journal of Human Genetics, 2, 111176.

Neale, M. C., \& Cardon, L. R. (1992). Methodology for genetic studies of twins and families. https://doi.org/10.1007/978-94-015-8018-2

Nila, S., Barthes, J., Crochet, P. A., Suryobroto, B., \& Raymond, M. (2018). Kin selection and male homosexual preference in Indonesia. Archives of Sexual Behavior, 47, 24552465. https://doi.org/10.1007/s10508-018-1202-y

Nishi, A., Alexander, M., Fowler, J. H., \& Christakis, N. A. (2020). Assortative mating at loci under recent natural selection in humans. BioSystems, 187, 104040. https://doi.org/10.1016/j.biosystems.2019.104040

Panksepp, J. (1998). Affective neuroscience: The foundations of human and animal. New York, NY: Oxford University Press.

Pearce, E., Wlodarski, R., Machin, A., \& Dunbar, R. I. M. (2019). Genetic Influences on Social Relationships: Sex Differences in the Mediating Role of Personality and Social Cognition. Adaptive Human Behavior and Physiology, 5, 331-351.

https://doi.org/10.1007/s40750-019-00120-5

Penke, L., \& Jokela, M. (2016). The evolutionary genetics of personality revisited. Current Opinion in Psychology, 7, 104-109. https://doi.org/10.1016/j.copsyc.2015.08.021

Plomin, R., \& Deary, I. J. (2015). Genetics and intelligence differences: Five special findings. Molecular Psychiatry, 20, 98-108. https://doi.org/10.1038/mp.2014.105

Plomin, R., DeFries, J. C., Knopik, V. S., \& Neiderhiser, J. M. (2016). Top 10 Replicated Findings From Behavioral Genetics. Perspectives on Psychological Science, 11(1), 323. https://doi.org/10.1177/1745691615617439

Polderman, T. J. C., Benyamin, B., De Leeuw, C. A., Sullivan, P. F., Van Bochoven, A., Visscher, P. M., \& Posthuma, D. (2015). Meta-analysis of the heritability of human traits based on fifty years of twin studies. Nature Genetics, 47(7), 702-709. https://doi.org/10.1038/ng.3285

Proulx, S. R., \& Østman, B. (2016). Natural Selection, Introduction to. Encyclopedia of Evolutionary Biology, 3, 100-103. https://doi.org/10.1016/B978-0-12-800049-6.000263

Rahman, Q., \& Wilson, G. D. (2003). Born gay? The psychobiology of human sexual orientation. Personality and Individual Differences, 34(8), 1337-1382.

Rantala, M. J., Luoto, S., Borráz-León, J. I., \& Krams, I. (2021). Bipolar disorder: An 
evolutionary psychoneuroimmunological approach. Neuroscience and Biobehavioral Reviews, 122, 28-37. https://doi.org/10.1016/j.neubiorev.2020.12.031

Rantala, M. J., Luoto, S., Krama, T., \& Krams, I. (2019). Eating disorders: An evolutionary psychoneuroimmunological approach. Frontiers in Psychology, 10, 2200. https://doi.org/10.3389/fpsyg.2019.02200

Rees, J. S., Castellano, S., \& Andrés, A. M. (2020). The genomics of human local adaptation. Trends in Genetics, 36(6), 415-428. https://doi.org/10.1016/j.tig.2020.03.006

Rietveld, C. A., Medland, S. E., Derringer, J., Yang, J., Esko, T., Martin, N. W., ... Koellinger, P. D. (2013). GWAS of 126,559 individuals identifies genetic variants associated with educational attainment. Science, 340, 1467-1471. https://doi.org/10.1126/science.1235488

Robinson, M. R., Kleinman, A., Graff, M., Vinkhuyzen, A. A. E., Couper, D., Miller, M. B., ... Nolte, I. M. (2017). Genetic evidence of assortative mating in humans. Nature Human Behaviour, 1.

Safran, R. J., Scordato, E. S. C., Symes, L. B., Rodríguez, R. L., \& Mendelson, T. C. (2013). Contributions of natural and sexual selection to the evolution of premating reproductive isolation: A research agenda. Trends in Ecology and Evolution, 28, 643-650. https://doi.org/10.1016/j.tree.2013.08.004

Segal, N. L. (2008). Born together - reared apart: The landmark Minnesota twin study. Cambridge, MA: Harvard University Press.

Segal, N. L. (2013). Personality similarity in unrelated look-alike pairs: Addressing a twin study challenge. Personality and Individual Differences, 54, 23-28. https://doi.org/10.1016/j.paid.2012.07.031

Segal, N. L., \& Macdonald, K. B. (1998). Behavioral genetics and evolutionary psychology: Unified perspective on personality research. Human Biology, 70, 159-184.

Slatkin, M. (2008). Linkage disequilibrium - Understanding the evolutionary past and mapping the medical future. Nature Reviews Genetics, 9, 477-485. https://doi.org/10.1038/nrg2361

Stoltzfus, A., \& Cable, K. (2014). Mendelian-mutationism: The forgotten evolutionary synthesis. Journal of the History of Biology, 47, 501-546. https://doi.org/10.1007/s10739-014-9383-2

Struik, D., Sanna, F., \& Fattore, L. (2018). The Modulating Role of Sex and AnabolicAndrogenic Steroid Hormones in Cannabinoid Sensitivity. Frontiers in Behavioral Neuroscience, 12. https://doi.org/10.3389/fnbeh.2018.00249

Stulp, G., \& Barrett, L. (2016). Evolutionary perspectives on human height variation. Biological Reviews, 91(1), 206-234. https://doi.org/10.1111/brv.12165

Swift-Gallant, A. (2019). Individual differences in the biological basis of androphilia in mice and men. Hormones and Behavior. https://doi.org/10.1016/j.yhbeh.2018.12.006

Swift-Gallant, A., Coome, L. A., Aitken, M., Ashley Monks, D., \& VanderLaan, D. P. (2019). Evidence for distinct biodevelopmental influences on male sexual orientation. Proceedings of the National Academy of Sciences of the United States of America. https://doi.org/10.1073/pnas.1809920116 
Syme, K. L., \& Hagen, E. H. (2020). Mental health is biological health: Why tackling "diseases of the mind" is an imperative for biological anthropology in the 21 st century. American Journal of Physical Anthropology, 171, 87-117. https://doi.org/10.1002/ajpa.23965

Tinbergen, N. (1963). On aims and methods of ethology. Zeitschrift Für Tierpsychologie, 20(4), 410-433. https://doi.org/10.1111/j.1439-0310.1963.tb01161.x

Tooby, J., \& Cosmides, L. (1990). On the universality of human nature and the uniqueness of the individual: The role of genetics and adaptation. Journal of Personality, 58, 17-67. https://doi.org/10.1111/j.1467-6494.1990.tb00907.x

Tucker-Drob, E. M., \& Bates, T. C. (2016). Large cross-national differences in gene $\times$ socioeconomic status interaction on intelligence. Psychological Science, 27, 138-149. https://doi.org/10.1177/0956797615612727

Uchiyama, R., Spicer, R., \& Muthukrishna, M. (2020). Cultural evolution of genetic heritability. BioRxiv. https://doi.org/10.1101/2020.06.23.167676

van der Zee, M. D., Helmer, Q., Boomsma, D. I., Dolan, C. V., \& de Geus, E. J. C. (2020). An Extended Twin-Pedigree Study of Different Classes of Voluntary Exercise Behavior. Behavior Genetics, 50, 94-104. https://doi.org/10.1007/s10519-019-09990-7

Van Doorn, G. S., Edelaar, P., \& Weissing, F. J. (2009). On the origin of species by natural and sexual selection. Science, 326, 1704-1707. https://doi.org/10.1126/science.1181661

Varella, M. A. C., Luoto, S., Soares, R. B. da S., \& Valentova, J. V. (2021). COVID-19 Pandemic on Fire: Evolved Propensities for Nocturnal Activities as a Liability Against Epidemiological Control. Frontiers in Psychology, 12, 646711. https://doi.org/10.3389/fpsyg.2021.646711

Wiley, R. H. (2020). Natural Selection. In Todd K Shackelford \& V. A. Weekes-Shackelford (Eds.), Encyclopedia of Evolutionary Psychological Science (pp. 1-12). https://doi.org/10.1007/978-3-319-16999-6_2095-1

Wilson, D. S. (1998). Adaptive individual differences within single populations. Philosophical Transactions of the Royal Society B: Biological Sciences, 353, 199-205. https://doi.org/10.1098/rstb.1998.0202

Winther, R. G. (2000). Darwin on variation and heredity. Journal of the History of Biology, 33, 425-455. https://doi.org/10.1023/A:1004834008068

Woodley, M. A. (2011). The cognitive differentiation-integration effort hypothesis: A synthesis between the fitness indicator and life history models of human intelligence. Review of General Psychology, 15, 228-245. https://doi.org/10.1037/a0024348

Woodley of Menie, M. A., Fernandes, H. B. F., \& Hopkins, W. D. (2015). The more gloaded, the more heritable, evolvable, and phenotypically variable: Homology with humans in chimpanzee cognitive abilities. Intelligence, 50, 159-163. https://doi.org/10.1016/j.intell.2015.04.002

Woodley of Menie, M. A., Kanazawa, S., Pallesen, J., \& Sarraf, M. A. (2020). Paternal age is negatively associated with religious behavior in a post-60s but not a pre-60s US birth cohort: Evidence for the Social Epistasis Amplification Model. Journal of Religion and Health, 59, 2733-2752. https://doi.org/10.1007/s10943-020-00987-9 
Woodley of Menie, M. A., Luoto, S., Peñaherrera-Aguirre, M., \& Sarraf, M. (2021). Life History is a major source of adaptive individual and species differences: A critical commentary on Zietsch and Sidari (2020). Evolutionary Psychological Science.

Woodley of Menie, M. A., Pallesen, J., \& Sarraf, M. A. (2018). Evidence for the Scarr-Rowe effect on genetic expressivity in a large US sample. Twin Research and Human Genetics, 21, 495-501. https://doi.org/10.1017/thg.2018.63

Woodley of Menie, M. A., Pawlik, P., Webb, M. T., Bruce, K. D., \& Devlin, P. F. (2019). Circadian leaf movements facilitate overtopping of neighbors. Progress in Biophysics and Molecular Biology, 146, 104-111. https://doi.org/10.1016/j.pbiomolbio.2018.12.012

Woodley of Menie, M. A., Peñaherrera-Aguirre, M., Dunkel, C., \& Sarraf, M. A. (2021). Evidence for the Scarr-Rowe effect on genetic expressivity in the Health and Retirement Study. Twin Research \& Human Genetics. https://doi.org/10.1017/thg.2021.13

Woodley of Menie, M. A., \& Sarraf, M. A. (2021). Controversies in evolutionary psychology. In T.K. Shackelford \& V. A. Weekes-Shackelford (Eds.), Encyclopedia of Evolutionary Psychological Science. https://doi.org/10.1007/978-3-319-16999-6_2175-1

Xia, C., Canela-Xandri, O., Rawlik, K., \& Tenesa, A. (2020). Evidence of horizontal indirect genetic effects in humans. Nature Human Behaviour. https://doi.org/10.1038/s41562020-00991-9

Yang, J., Lee, S. H., Goddard, M. E., \& Visscher, P. M. (2011). GCTA: A tool for genomewide complex trait analysis. American Journal of Human Genetics, 88, 76-82. https://doi.org/10.1016/j.ajhg.2010.11.011

Young, E. S., Frankenhuis, W. E., \& Ellis, B. J. (2020). Theory and measurement of environmental unpredictability. Evolution and Human Behavior, 41, 550-556. https://doi.org/10.1016/j.evolhumbehav.2020.08.006

Zietsch, B. P., de Candia, T. R., \& Keller, M. C. (2015). Evolutionary behavioral genetics. Current Opinion in Behavioral Sciences, 2, 73-80. https://doi.org/10.1016/j.cobeha.2014.09.005

Zietsch, B. P., Sidari, M. J., Murphy, S. C., Sherlock, J. M., \& Lee, A. J. (2020). For the good of evolutionary psychology, let's reunite proximate and ultimate explanations. Evolution and Human Behavior. https://doi.org/10.1016/j.evolhumbehav.2020.06.009 